\title{
Effectiveness of trastuzumab as adjuvant therapy in patients with early stage breast cancer: A systematic review and meta-analysis
}

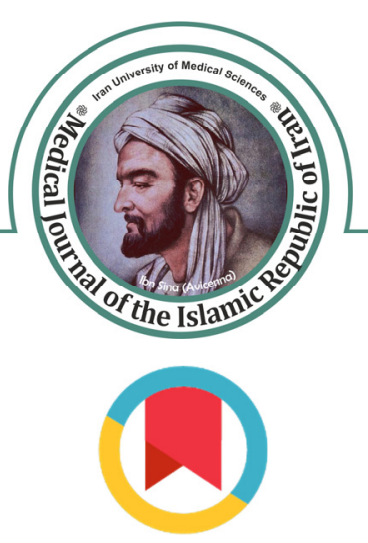

\author{
Majid Davari ${ }^{1}$, Bahman Amani², Fariborz Mokarian ${ }^{3}$, Mohsen Hoseini ${ }^{4}$, Arash Akbarzadeh ${ }^{5}$, \\ Nastaran Heidarzadeh Khoramabadi ${ }^{6 *}$
}

Received: 20 Aug 2017

Published: 16 Dec 2017

\section{Abstract}

Background: Trastuzumab in combination with chemotherapy has long been established as a standard treatment for HER2-positive patients in early stage breast cancer (BC). The present study aimed at assessing the effectiveness of trastuzumab adjuvant therapy in early stage BC in overall survival (OS) and disease-free survival (DFS).

Methods: A systematic review and meta-analysis was performed to evaluate the effectiveness of trastuzumab adjuvant therapy. PubMed, Cochrane library, Scopus, Web of Science, and Embase databases were searched for relevant RCTs from the beginning to February 2017. Quality assessment of studies was conducted using the Cochrane Risk of Bias Tool. The desired outcomes were OS and DFS.

Results: A total of 1818 articles were identified first, however, only 11 studies were eligible to be included in this study. Our findings and meta-analysis results revealed that trastuzumab is effective in increasing OS (OS hazard ratio: $-0.286 \pm 0.049,95 \% \mathrm{CI}(-0.381$, $0.191)$ ) and improving DFS (DFS hazard ratio: $-0.419 \pm 0.077,95 \%$ CI $(-0.569,-0.269))$. The most serious but negligible side effect of trastuzumab is congestive heart failure.

Conclusion: Adding trastuzumab as adjuvant therapy in early stages of BC in HER2 positive patients could increase OS and DFS of the patients effectively.

Keywords: Trastuzumab, Breast Cancer, Herceptin, Meta-analysis, Adjuvant Therapy

Copyright@ Iran University of Medical Sciences

Cite this article as: Davari M, Amani B, Mokarian F, Hoseini M, Akbarzadeh A, Heidarzadeh Khoramabadi N. Effectiveness of trastuzumab as adjuvant therapy in patients with early stage breast cancer: A systematic review and meta-analysis. Med J Islam Repub Iran. 2017(16 Dec);31:88. https://doi.org/10.14196/mjiri.31.88

\section{Introduction}

Breast cancer $(\mathrm{BC})$ is the most common type of cancer worldwide and is one of the leading causes of death in cancer patients. Although the rate of $\mathrm{BC}$ is decreasing in developed countries, it is rapidly increasing in developing countries $(1,2)$. Statistics show that every year more than one million new cases of cancer are diagnosed in the world and more than 400000 people are lost to cancer (3). Early diagnosis and treatment of $\mathrm{BC}$ in its early stages could significantly increase the survival rate of $\mathrm{BC}$ patients (4). Trastuzumab in combination with chemotherapy has been

Corresponding author: Dr Nastaran Heidarzadeh Khoramabadi, nastaran.heidarzadeh@gmail.com

1. Department of Pharmacoeconomics and Pharmaceutical Administration, Faculty of Pharmacy, Tehran University of Medical Sciences, Tehran, Iran, \& Pharmaceutical Management and Economics Research Center, Tehran University of Medical Sciences, Tehran, Iran.

2. Department of Health Management and Economics, School of Public Health, Tehran University of Medical Sciences, Tehran, Iran.

3. Breast cancer Research center, Isfahan University of Medical Science, Isfahan, Iran.

4. Department of Biostatistics and Epidemiology, School of Health, Isfahan University of Medical Sciences, Isfahan, Iran.

5. Department of Epidemiology and Biostatistics, School of Public Health, Tehran University of Medical Sciences, Tehran, Iran.

6. Department of Drug and Food Control, School of Pharmacy, Tehran University of Medical Sciences, Tehran, Iran. confirmed as the standard treatment of BC for HER2 positive patients (5). Many studies have found that trastuzumab in combination with chemotherapy prolongs overall survival (OS) (6) and improves progression-free survival (PFS) (7). Women with HER2 positive breast cancer are at greater risk for progression of disease and death compared to women who are not HER2 positive $(8,9)$.

Thus, the purpose of treatment strategies of this type of cancer is blocking HER2 positive BC. Trastuzumab is a recombinant human monoclonal antibody that binds to the

$\uparrow$ What is "already known" in this topic:

Previous studies confirmed that trastuzumab is effective in improving overall survival of the patients with breast cancer.

\section{$\rightarrow$ What this article adds:}

The results of new trials were added to this systematic review. The results of the current meta-analysis showed the quantitative effect of trastuzumab in improving overall survival and progression of free survival in patients with breast cancer. 
chain extracellular protein HER2 and is a key component in treatment of HER2 positive BC in its early stages (10).

The present study aimed at evaluating the effectiveness of trastuzumab in the treatment of early stage BC.

\section{Methods}

\section{Literature search}

A systematic review and meta-analysis was conducted to evaluate the effectiveness of trastuzumab using updated data. PubMed, Cochrane library, Web of Science, Embase, and Google Scholar databases were searched from the beginning to February 2017. The reference list of the selected RCTs and reviews were also searched for additional citations.

\section{Structured questions}

Our focus was on studies that evaluated the results of treatment in women with early BC. Our interested intervention was trastuzumab as adjuvant therapy in comparison with basic treatment without trastuzumab. Overall survival (OS) and disease- free survival (DFS) were considered as desired outcomes. Randomized controlled trials (RCTs) were the preferred study design. Studies that reported the results of monotherapy of the trastuzumab in BC were excluded. Observational, experimental, and animal studies were also excluded.

All selected studies were saved in Endnote library. After removing duplications, titles and abstracts were screened independently by 2 authors. The disagreements between the 2 authors were resolved by discussion.
Breast Neoplasms[Mesh]

Breast [Title/Abstract] AND (cancer* [Title/Abstract] OR tumour*[Title/Abstract] OR tumor*[Title/Abstract] OR neoplas*[Title/Abstract])

Trastuzumab or Herceptin [Title/Abstract]

1 OR 2

3 And 4 Filters: Clinical trial; Humans

\section{Quality appraisal method}

The quality of the selected studies was evaluated using the Cochrane Risk of Bias Tool and Jadad scale.

\section{Data analysis}

A meta-analysis was conducted to compare the effectiveness of trastuzumab as adjuvant therapy in early stage BC. This analysis was performed using the National Council for Social Studies (NCSS) Version 11 software. Heterogeneity was evaluated using Cochran`s $\mathrm{Q}$ test and I2 criteria. I2 equal to $50 \%$ indicated low heterogeneity. Inverse variance method was used to weight the studies.

\section{Results}

\section{Selection of studies}

The total of 5 databases were searched and 1818 articles were detected primarily. Following the screening of the titles and abstracts, 87 full text studies were selected. However, only 11 studies met the inclusion criteria (11-21), all of which were RCTs. Figure 1 demonstrates the preferred reporting items for systematic reviews and meta-analyses (PRISMA) diagram of the selection process.

\section{Search strategy}

Our search strategy for PubMed is presented as follows:

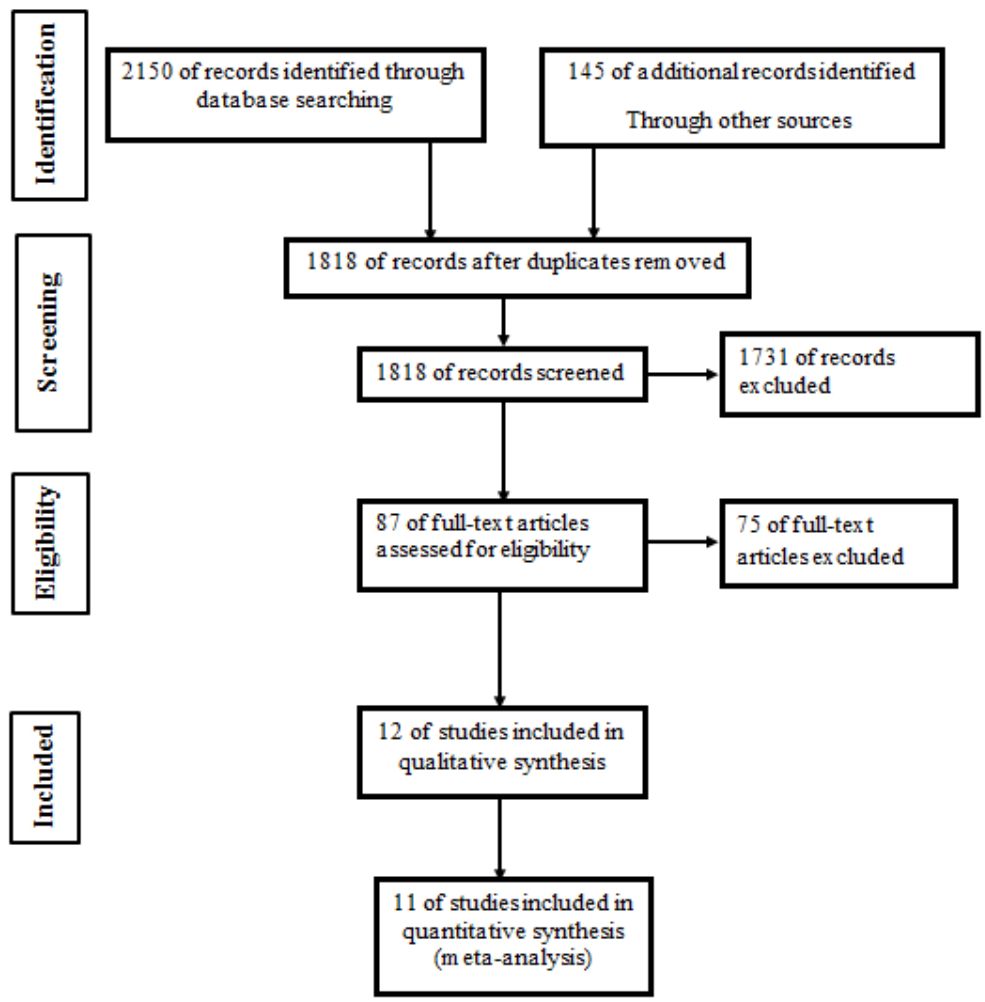

Fig. 1. PRISMA diagram of study selection 


\begin{tabular}{|c|c|c|c|c|c|c|}
\hline No. & Study & $\mathrm{N}$ & Duration & Intervention & Comparison & Jadad scale \\
\hline 1 & $\mathrm{~B} 31(10)$ & 394 & $>6$ months & Trastuzumab & Without Trastuzumab & 2 \\
\hline 2 & BCIRG006(11) & 1703 & $>6$ months & Trastuzumab & Without Trastuzumab & 1 \\
\hline 3 & Buzdar(12) & 42 & 6 months & Trastuzumab & Without Trastuzumab & 3 \\
\hline 4 & Finher(13) & 1010 & $<6$ months & Trastuzumab & Without Trastuzumab & 3 \\
\hline 5 & Hera(14) & 5081 & $>6$ months & Trastuzumab & Without Trastuzumab & 3 \\
\hline 6 & Noah(15) & 235 & $>6$ months & Trastuzumab & Without Trastuzumab & 3 \\
\hline 7 & Pacs-04(16) & 528 & $>6$ months & Trastuzumab & Without Trastuzumab & 1 \\
\hline 8 & Goldhirsch(17) & 5102 & $>6$ months & Trastuzumab & Without Trastuzumab & 3 \\
\hline 9 & Joensuu(18) & 1500 & $<6$ months and $>6$ months & Trastuzumab & Chemotherapy without Trastuzumab & 2 \\
\hline 10 & Schneider(19) & 227 & $<6$ months and $>6$ months & Trastuzumab & 1 year or 12 weeks Trastuzumab & 3 \\
\hline 11 & Gianni(20) & 5102 & $>6$ months & Trastuzumab & Without trasuzumab & 3 \\
\hline \multicolumn{7}{|c|}{ Table 2. The results of the meta-analysis of OS outcome } \\
\hline \multicolumn{3}{|c|}{ Study } & $\operatorname{Ln}(H R)$ & $\operatorname{SE}(\operatorname{Ln}(H R))$ & Confidence interval & Weight \\
\hline \multicolumn{3}{|c|}{ B31 } & -0.4 & 0.17 & $(-0.733,-0.067)$ & 8.16 \\
\hline \multicolumn{3}{|c|}{ BCIRG006 } & -0.46 & 0.13 & $(-0.715,-0.205)$ & 13.95 \\
\hline \multicolumn{3}{|c|}{ Finher } & -0.6 & 0.36 & $(-1.3,0.106)$ & 1.82 \\
\hline \multicolumn{3}{|c|}{ Hera } & -0.46 & 0.17 & $(-0.793,-0.127)$ & 8.16 \\
\hline \multicolumn{3}{|c|}{ Noah } & -0.48 & 0.3 & $(-1.07,0.108)$ & 2.62 \\
\hline \multicolumn{3}{|c|}{ Pacs-04 } & 0.24 & 0.32 & $(-0.387,0.867)$ & 2.3 \\
\hline \multicolumn{3}{|c|}{ Goldhirsch } & -0.274 & 0.079 & $(-0.429,-0.120)$ & 37.96 \\
\hline \multicolumn{3}{|c|}{ Schneider } & 0.336 & 0.325 & $(-0.299,0.973)$ & 2.24 \\
\hline \multicolumn{3}{|c|}{ Gianni } & -0.162 & 0.102 & $(-0.362,0.037)$ & 22.79 \\
\hline \multicolumn{3}{|c|}{ Overall effect } & -0.286 & 0.049 & $(-0.381,-0.191)$ & \\
\hline \multicolumn{3}{|c|}{ Directional Zero-Effect Test } & $\chi^{2}=34.689, d f=1, P-v a l$ & lue $=0.000$ & & \\
\hline
\end{tabular}

Total number of 20924 patients were included in the selected studies, most of them followed- up the patients for more than 6 months. The summary of the characteristics of the studies are presented in Table 1.

\section{The OS results}

The results of the Cochran's Q test revealed that the homogeneity between selected studies was appropriate (Cochran`s $\mathrm{Q}=12.3437, \mathrm{I} 2=0.35 \%$ ), presented in the Galbraith plot. This plot shows that all studies are placed within the confidence interval of the regression line of Galbraith plot, and thus confirm that the homogeneity of the studies is sufficient. When the homogeneity criterion of the studies was met, the fixed model was used to combine the results of OS (Fig. 2).

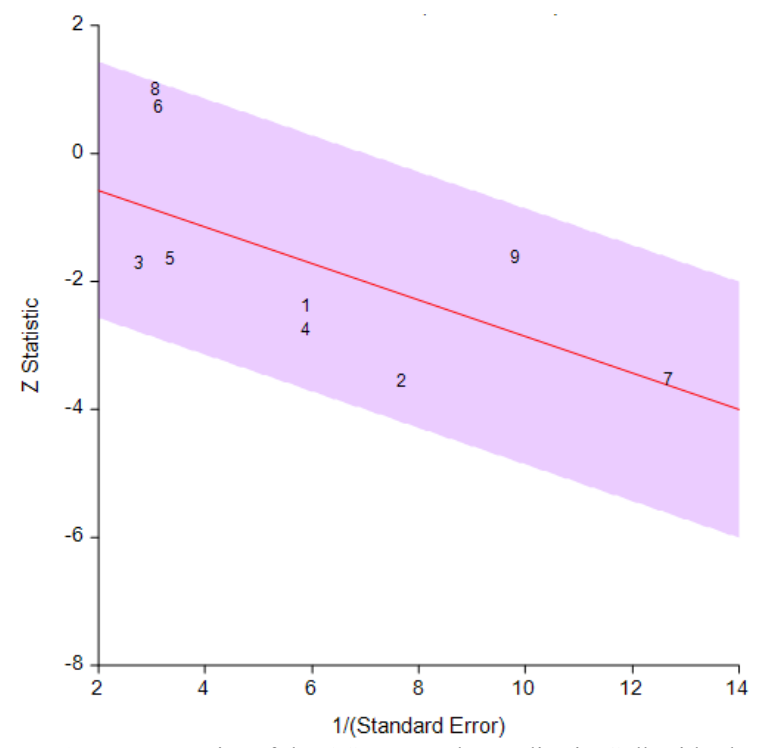

Fig. 2. Heterogeneity of the OS among the studies in Galbraith plot
The results of the relative weight of the studies showed that all studies had reported statistically similar effects with nonzero results. The significance level of chi square test was less than 0.05 . Moreover, the logarithm of the hazard ratio for "moderate the impact of" was less than zero, indicating that the hazard ratio was between 0 and 1 (Table 2). Therefore, it is concluded that trastuzumab is effective in increasing patient survival (Fig. 3).

\section{The DFS Results}

The results of the Cochran's Q test indicated that homogeneity was not met among the selected studies(Cochran's $\mathrm{Q}=32.9034, \mathrm{I} 2=0.69 \%$ ). Therefore, the random model was applied to aggregate the results of DFS. Figure 4 demonstrates the heterogeneity of DFS within the selected studies with Galbraith plot.

The results in Table 3 clarify that the significance level of chi square test was less than 0.05 , and thus it is concluded

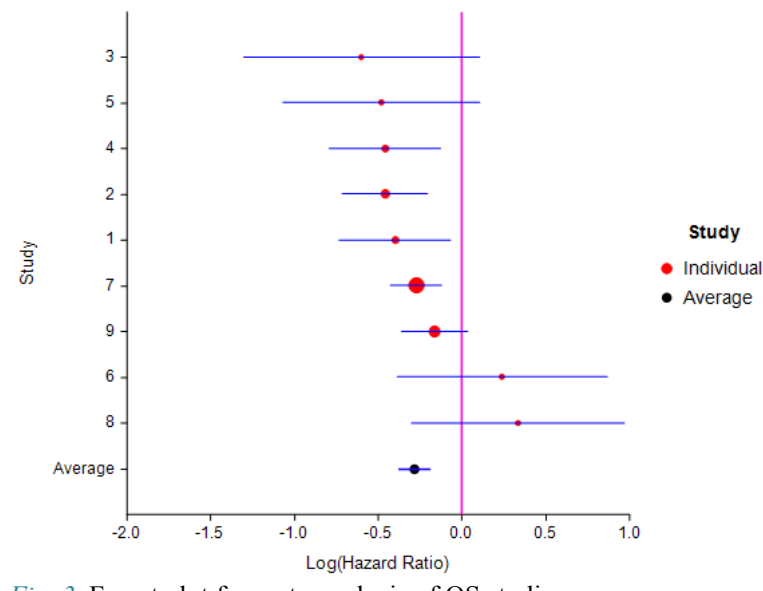

Fig. 3. Forest plot for meta-analysis of OS studies 


\begin{tabular}{|c|c|c|c|c|}
\hline Study & $\operatorname{Ln}(H R)$ & $S E(\operatorname{Ln}(H R))$ & Confidence interval & Weight \\
\hline B31 & -0.73 & 0.11 & $(-0.946,-0.514)$ & 12.49 \\
\hline Bcirg006 & -0.45 & 0.1 & $(-0.646,-0.254)$ & 13.07 \\
\hline Buzdar & -2.27 & 1.11 & $(-4.44,-0.094)$ & 0.462 \\
\hline Finher & -0.87 & 0.35 & $(-1.56,-0.184)$ & 3.72 \\
\hline Hera & -0.46 & 0.09 & $(-0.636,-0.284)$ & 13.65 \\
\hline Noah & -0.53 & 0.22 & $(-0.961,-0.099)$ & 7.04 \\
\hline Pacs-04 & -0.15 & 0.18 & $(-0.503,0.203)$ & 8.72 \\
\hline Goldhirsch & -0.274 & 0.07 & $(-0.412,-0.137)$ & 14.75 \\
\hline Joensuu & -0.892 & 0.271 & $(-1.423,-0.514)$ & 5.51 \\
\hline Schneider & 0.262 & 0.253 & $(-0.234,0.758)$ & 5.93 \\
\hline Gianni & -0.274 & 0.07 & $(-0.412,-0.137)$ & 14.75 \\
\hline Overall effect & -0.419 & 0.077 & $(-0.569,-0.269)$ & \\
\hline Directional Zero-Effect Test & \multicolumn{3}{|c|}{$\chi^{2}=116.915, d f=1, P-$ value $=0.000$} & \\
\hline
\end{tabular}

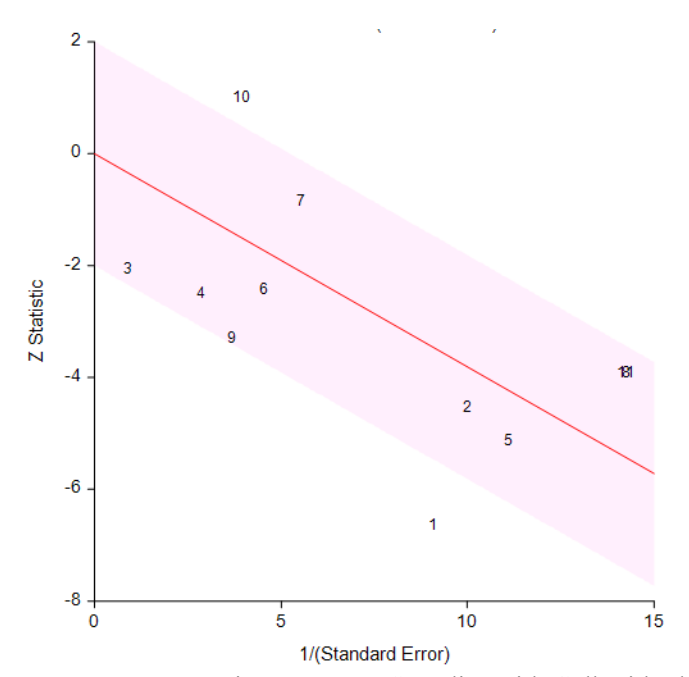

Fig. 4. Heterogeneity among DFS studies with Galbraith plot

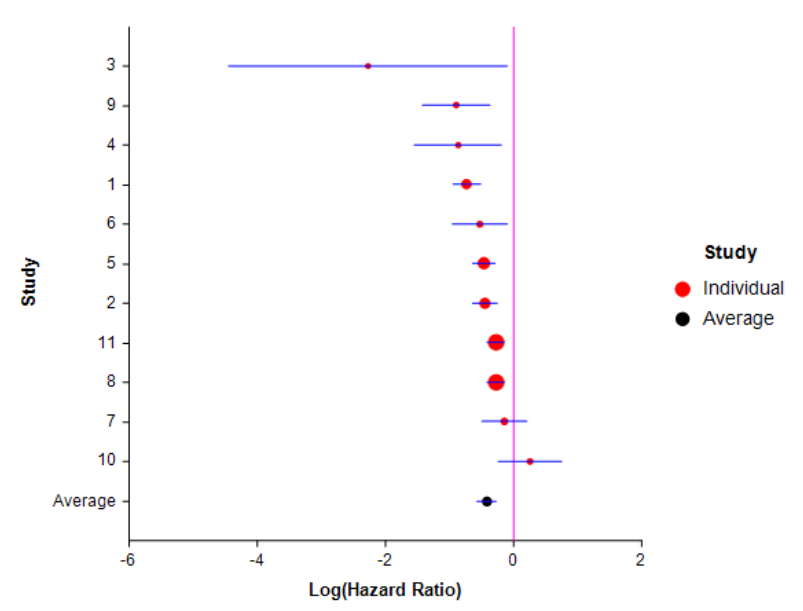

Fig. 5. Forest plot for meta-analysis of DFS studies that trastuzumab as adjuvant therapy is effective in improving DFS (Fig. 5).

Risk of bias results is presented in Table 4.

\section{Discussion}

The present study aimed at evaluating the effectiveness of trastuzumab on OS and DFS as adjuvant therapy in the treatment of early stage BC. The primary results of RCTs revealed that the hazard ratio (HR) of OS in trastuzumab protocol was significantly lower than ordinary chemotherapy. However, Buzdar study reported a HR equal to 1, with no difference between the 2 treatment groups. Maximum HR was reported in Schneider B.P study $(\mathrm{HR}=1.4)$, and minimum HR was related to FinHer study $(\mathrm{HR}=0.55)$.

The primary results of RCTs revealed a significant in-

\begin{tabular}{lcccc}
\multicolumn{2}{l}{ Table 4. The summary of risk of BIAS results } & & \\
\hline Study & Sequence generation & Allocation concealment & $\begin{array}{c}\text { Blinding of participants } \\
\text { Incomplete outcome } \\
\text { data }\end{array}$ & $\begin{array}{c}\text { Selective outcome } \\
\text { reporting }\end{array}$ \\
\hline B31 & Low & Unclear & High & High \\
BCIRG006 & Unclear & Unclear & Unclear \\
Buzdar & Low & Low & High \\
FinHer & Low & Low & Low \\
HERA & Low & Unclear & High & Low \\
NOAH & Low & Unclear & High & High \\
PACS-04 & Unclear & Unclear & High & Low \\
Gianni & Low & Unclear & High & Low \\
Goldhirsch & Low & Unclear & Unclear \\
Joensuu & Low & Unclear & High & Low \\
Schneider & Low & Unclear & High & Low \\
\hline
\end{tabular}

$4 \quad$ http://mjiri.iums.ac.ir 
crease in DFS for trastuzumab adjuvant therapy. Scheneider study, with $\mathrm{HR}=1.3$, has reported the highest effectiveness rate; and Buzdar study, with $\mathrm{HR}=0.103$, showed the lowest effectiveness rate for trastuzumab adjuvant therapy. The number of patients in RCTs varied from 42 to 5102 .

This meta-analysis was conducted to determine the relative weights of various studies. We combined the HRs and the number of patients in the studies to obtain more accurate and reliable results. We meta-analyzed the OS with 9 RCTs and DFS with 11 RCTs.

Considering the results of the relative weight of the selected studies and HR, Goldhirsch study reported the maximum influence on the OS of the patients, with numerical value of 28.85 . The second best results belonged to Luca Gianni study, with the numerical value of 19.37. FinHer study, with the value of 0.999 , had the lowest effectiveness in this regard. Buzdar study, with $\mathrm{HR}=1$, showed that the rate of OS with and without trastuzumab is the same in $\mathrm{BC}$ patients.

When analyzing DFS, we found that Luca Gianni and Goldhirsch studies, with the value of 11.21, reported the maximum effectiveness on DFS. The lowest effectiveness was shown in Buzdar study, with the value of 0.047. Thus, it is concluded that trastuzumab is significantly effective in improving DFS.

The most serious side effect of trastuzumab was improvement of congestive heart failure (CHF) (24), but some studies stated that the risk of CHF for $\mathrm{BC}$ patients could be considered zero (26). Furthermore, the results of studies showed that if cardiac performance is monitored during the treatment time, the damage would not be life- threating and is allowed to continue (24). Also, it is recommended that trastuzumab be added only to anthracycline-free chemotherapy regimens (25).

The FinHer study ( 9 weeks treatment with trastuzumab) demonstrated acceptable and appropriate effectiveness in increasing OS and DFS (26), however, when considering the low relative weight of the study, it is clear that the results would not be confident enough and could not be considered proportional to the total BC population.

In summary, the main findings of this meta-analysis support and update the results of previous studies and confirm that trastuzumab is effective in improving OS and DFS in $\mathrm{BC}$ patients. These findings could help healthcare providers to prescribe effective medication for their patients. Likewise, policy makers could use these results as a primary data to provide better coverage for BC patients. Moreover, complementary data would be the results of cost-effectiveness analysis.

\section{Conclusion}

The addition of trastuzumab as adjuvant therapy in early stages of BC in HER2 positive patients could increase OS and DFS of the patients effectively. The result of FinHer study encourage conducting further studies on this treatment protocol to manage $\mathrm{BC}$.

\section{Recommendations for the future}

Considering the shorter treatment period of FinHer study compared to other studies and because of lower costs and lower side effects, it is strongly recommended that larger trials be conducted in this field.

\section{Conflict of Interests}

The authors declare that they have no competing interests.

\section{References}

1. Jemal A, Siegel R, Xu J, Ward E. Cancer statistics, 2010. CA cancer J Clin. 2011Mar-Apr; 61(2):133-4.

2. Geyer CE, Forster J, Lindquist D, Chan S, Romieu CG, Pienkowski T, et al. Lapatinib plus capecitabine for HER2- positive Advanced Breast Cancer. N Engl J Med. 2006 Dec 28;355(26):2733-43.

3. Ferley J, Bray F, Sankila R, Parkin DM. EUCAN: Cancer incidence. Mortality and prevalence in the European Union 1998. IARC Cancer Base No.4, Version 5.0. Lyon: IARC Press. 1999.

4. Corle DK, Sharbaugh C, Mateski DJ, Coyne T, Paskett ED, Cahill J. Self related quality of life measures: Effect of change to a low fat, highfiber, fruit and vegetable enriched diet. Ann Behav Med. August 2001;23(3):198-207.

5. Romond EH, Perez EA, Bryant J, Suman VJ, Geyer CE Jr, Davidson NE, et al. Trastuzumab plus adjuvant chemotherapy for operable HER2 positive breast cancer. N Engl J Med. 2005 Oct 20:353(16):1673-83.

6. Perez EA, Romond EH, Suman VJ, Jeong JH, Davidson NE, Geyer CE $\mathrm{Jr}$, et al. Four- year follow up trastuzumab plus adjuvant chemotherapy for operable epidermal growth factor receptor 2- positive breast cancer: joint analysis of from NCCTG N9831 and NSABP B-31. J Clin Oncol. 2011 Sep 1;29(25):3366-73.

7. Goldhirsch A, Gelber RD, Piccart-Gebhart MJ, de Azambuja E, Procter M, Suter TM, et al. 2 years versus 1 year of adjuvant trastuzumab for HER2-positive breast cancer (HERA): an open-label, randomised controlled trial. Lancet. 2013 Sep 21;382(9897):1021-8

8. Gianni L, Eiermann W, Semiglazov V, Manikhas A, Lluch A, Tjulandin S, et al. Neoadjuvant chemotherapy with trastuzumab followed by adjuvant trastuzumab versus neoadjuvant chemotherapy alone, in patients with HER2-positive locally advanced breast cancer (the NOAH trial): a randomized controlled superiority trial with a parallel HER2-negative cohort. Lancet. 2010 Jan 30;375(9712):377-84.

9. Slamon DJ, Clark GM, Wong SG, Levin WJ, Ullrich A, McGuire WL. Human breast cancer: correlation of relapse and survival with amplification of the HER-2/neu oncogene. Science. 1987 Jan 9;235(4785):177-82.

10. Bartsch R, Wenzel C, Steger GG. Trastuzumab in the management of early and advanced stage breast cancer. Biologics. 2007 Mar;1(1): 1931

11. Romond EH, Perez EA, Bryant J, Suman VJ, Geyer CE Jr, Davidson NE, et al. Trastuzumab plus adjuvant chemotherapy for operable HER2-positive breast cancer. N Engl J Med. 2005 Oct 20;353(16):1673-84

12. Smith I, Procter M, Gelber RD, Guillaume S, Feyereislova A, Dowsett M, et al. 2-year follow-up of trastuzumab after adjuvant chemotherapy in HER2-positive breast cancer: a randomised controlled trial. Lancet. 2007 Jan 6;369(9555):29-36.

13. Buzdar AU, Valero V, Ibrahim NK, Francis D, Broglio KR, Theriault RL, et al. Neoadjuvant therapy with paclitaxel followed by 5 -fluorouracil, epirubicin, and cyclophosphamide chemotherapy and concurrent trastuzumab in human epidermal growth factor receptor 2-positive operable breast cancer: an update of the initial randomized study population and data of additional patients treated with the same regimen. Clin Cancer Res. 2007 Jan 1;13(1):228-33.

14. Joensuu H, Kellokumpu-Lehtinen PL, Bono P, Alanko T, Kataja V, Asola R, et al. Adjuvant docetaxel or vinorelbine with or without trastuzumab for breast cancer. N Engl J Med. 2006 Feb 23;354(8):80920.

15. Gianni L, Eiermann W, Semiglazov V, Manikhas A, Lluch A, Tjulandin S, et al. Neoadjuvant chemotherapy with trastuzumab followed by adjuvant trastuzumab versus neoadjuvant chemotherapy alone, in patients with HER2-positive locally advanced breast cancer (the NOAH trial):a randomised controlled superiority trial with a parallel HER2-negative cohort. Lancet. 2010;375:377-84.

16. Spielmann M, Rochè H, Deloizer T, Canon J, Romieu G, Bourgeois 
$\mathrm{H}$, et al. Trastuzumab for patients with axillarynode-positive breast cancer: results of the FNCLCC-PACS 04 trial. Journal of Clinical Oncology 2009;27(36):6129-34.

17. Goldhirsch A, Gelber RD, Piccart-Gebhart MJ, Azambuja E, Procter M, Suter TM, et al. 2 years versus 1 year of adjuvant trastuzumab for HER2-positive breast cancer (HERA): an open-label, randomised controlled trial. Lancet. 2013;382(9897):1021-8.

18. Joensuu H, Kellokumpu-Lehtinen PL, Huovinen R, Jukkola-Vuorine A, Tanner M, Kokko, R, et al. Outcome of patients with HER2-positive breast cancer treated with or without adjuvant trastuzumab in the Finland Capecitabine Trial (FinXX). Acta Oncologica. 2014;53(2):186194.

19. Schneider BP, O'Neill A, Shen F, Sledge GW, Thor AD, Kahanic SP, et al. Pilot trial of paclitaxel-trastuzumab adjuvant therapy for early stage breast cancer: a trial of the ECOG-ACRIN cancer research group (E2198). Br J Cancer. 2015;113(12):1651-7.

20. Gianni L, Dafni U, Gelber RD, Azambuja E, Muehlbauer S, Goldhirsch A, et al. Treatment with trastuzumab for 1 year after adjuvant chemotherapy in patients with HER2-positive early breast cancer: a 4year follow-up of a randomised controlled trial. Lancet Oncol. 2011;12:236-44.

21. Dahabreh IJ, Linardou H, Siannis F, Fountzilas G, Murray S. Trastuzumab in the adjuvant treatment of early- stage breast cancer: a systematic review and meta- analysis of randomized controlled trials. The Oncologist. 2008;13:620-630.

22. Viani GA, Afonso SL, Stefano EJ, Fendi LI, Soares FV. Adjuvant trastuzumab in the treatment of HER2-positive early breast cancer: a meta-analysis of published randomized trials. BMC Cancer. 2007; 7:1530.

23. Long HD, Lin YE, Zhang JJ, Zhong WZ, Zheng RN. Risk of Congestive Heart Failure in Early Breast Cancer Patients Undergoing Adjuvant Treatment with Trastuzumab: A Meta-Analysis. The Oncologist. 2016;21(5):547-554.

24. Tao C, Tao X, Yang L, Chun L, Juxiang C, Yicheng L, et al. Risk of cardiac dysfunction with trastuzumab in breast cancer patients: A metaanalysis. Cancer Treatment Reviews. 2011;37(4):312-320.

25. Dedes KJ, Szucs TD, Imesch P, Fedier A, Fehr MK, Fink D. Costeffectiveness of trastuzumab in the adjuvant treatment of early breast cancer: a model- based analysis of the HERA and FinHer trial. Annals of Oncoloy. 2007;18(9):1493-1499.

26. Moja L, Tagliabue L, Balduzzi S, Parmelli E, Pistotti V, Guarneri V, et al. Trastuzumab containing regimens for early breast cancer. Cochrane Database Syst Rev. 2012 Apr;18(4):CD006243. 